\title{
Unwarping of Unidirectionally Distorted EPI Images
}

\author{
Jan Kybic ${ }^{\dagger}$, Philippe Thévenaz, Arto Nirkko and Michael Unser
}

\begin{abstract}
Echo-planar imaging (EPI) is a fast nuclear magnetic resonance imaging method. Unfortunately, local magnetic field inhomogeneities induced mainly by the subject's presence cause significant geometrical distortion, predominantly along the phase-encoding direction, which must be undone to allow for meaningful further processing. So far, this aspect has been too often neglected.

In this paper, we suggest a new approach using an algorithm specifically developed for the automatic registration of distorted EPI images with corresponding anatomically correct MRI images. We model the deformation field with splines, which gives us a lot of flexibility while comprising the affine transform as a special case. The registration criterion is least-squares. Interestingly, the complexity of its evaluation does not depend on the resolution of the control grid. The spline model gives us good accuracy thanks to its high approximation order. The short support of splines leads to a fast algorithm. A multiresolution approach yields robust ness and additional speed-up.

The algorithm was tested on real as well as synthetic data, and the results were compared with a manual method. A wavelet-based Sobolev-type random deformation generator was developed for testing purposes. A blind test indicates that the proposed automatic method is faster, more reliable, and more precise than the manual one.
\end{abstract}

Keywords - image registration, splines, geometrical distortion, unwarping

\section{INTRODUCTION}

\section{A. EPI features}

Echo planar imaging (EPI) [1] is a fast magnetic resonance imaging (MRI) technique permitting an acquisition of a two-dimensional slice using a single excitation, which leads to very short scan times. It is used mainly for functional imaging (fMRI), the in vivo non-invasive study of the temporal, spatial and behavioral dependencies of brain activations. The basis of fMRI lies in the fact that deoxyhemoglobin (the hemoglobin without a bound oxygen molecule) is paramagnetic. Neural activation in the cerebral cortex leads to an increase of blood flow, hence to a decrease of deoxyhemoglobin concentration. ${ }^{1}$ This results in a measurable alteration of the magnetic field and in a consequent increase of signal intensity in the appropriately weighted MRI images (blood oxygen-level dependent, BOLD). It is therefore difficult to compensate for the unwanted magnetic field inhomogeneities induced mainly by the spatially varying magnetic susceptibility of the subject [2]. In contrast to conventional MRI, where the number

$\dagger$ indicates corresponding author. Jan Kybic, Philippe Thévenaz, and Michael Unser are with Biomedical Imaging Group, DMT/IOA, Swiss Federal Institute of Technology Lausanne, CH-1015 Lausanne EPFL, Switzerland, email: Jan.Kybic@epfl.ch. Arto Nirkko is with Inselspital, Bern, Switzerland

${ }^{1}$ This effect prevails over the increase of oxygen consumption. of excitations per slice is equal to the number of scan lines, in EPI the magnetic field gradients have to encode two coordinates simultaneously in one excitation. As one of the gradients (the so-called phase-encoding gradient) is several orders of magnitude weaker than the other, the inhomogeneous magnetic field will manifest itself mainly as a geometrical distortion of the 2D slice image along the direction of this gradient. This effect is clearly visible in Figure 1. Since the stronger gradient is less affected, the distortion is essentially unidirectional. Letting $g$ be the unknown warping (deformation) function, we have

$$
f^{o}(g(x, y), y) \simeq f^{u}(x, y)
$$

where $f^{o}$ is the observed EPI image and $f^{u}$ is the hypothetical ideal undistorted EPI image. We can consider each slice separately, as the shift in the $z$ axis due to patient's movement is insignificant because his head is attached. Should there be such a displacement, it can by readily corrected by existing algorithms [3].

\section{B. The reasons to unwarp}

The amplitude of the deformation $g$ can be as large as 3-5 mm [4] (confirmed by our own observations), which typically amounts to several pixels. In some cases, as in Figure 1, specifically intended to illustrate EPI distortion, the deformation can be even more pronounced. Moreover, $g$ can vary significantly from slice to slice and from acquisition to acquisition. For localization applications like stereotactic surgery, this inaccuracy is much larger than the required limit of $1 \mathrm{~mm}$ and therefore EPI cannot currently be used to this end. It also severely hinders the performance of the statistical processing of sets of fMRI images used to obtain activation information. Since the task-induced signal changes represent typically only $5-10 \%$ of the mean signal intensity in fMRI $[1,5]$, they will not stand out clearly unless the perturbations caused by the deformation $g$ are undone.

\section{Existing distortion correction techniques}

One approach consists in changing the acquisition procedure $[2,4,6]$. However, this is often not practical due to technical or organizational limitations, for example lack of support or approval. Furthermore, while the alternative acquisition sequences reduce the distortion, the distortion is never removed completely, and the methods usually sacrifice either sensitivity or acquisition speed.

The second group of methods uses a two-step procedure $[4,7]$. First, a field map or a deformation map is 


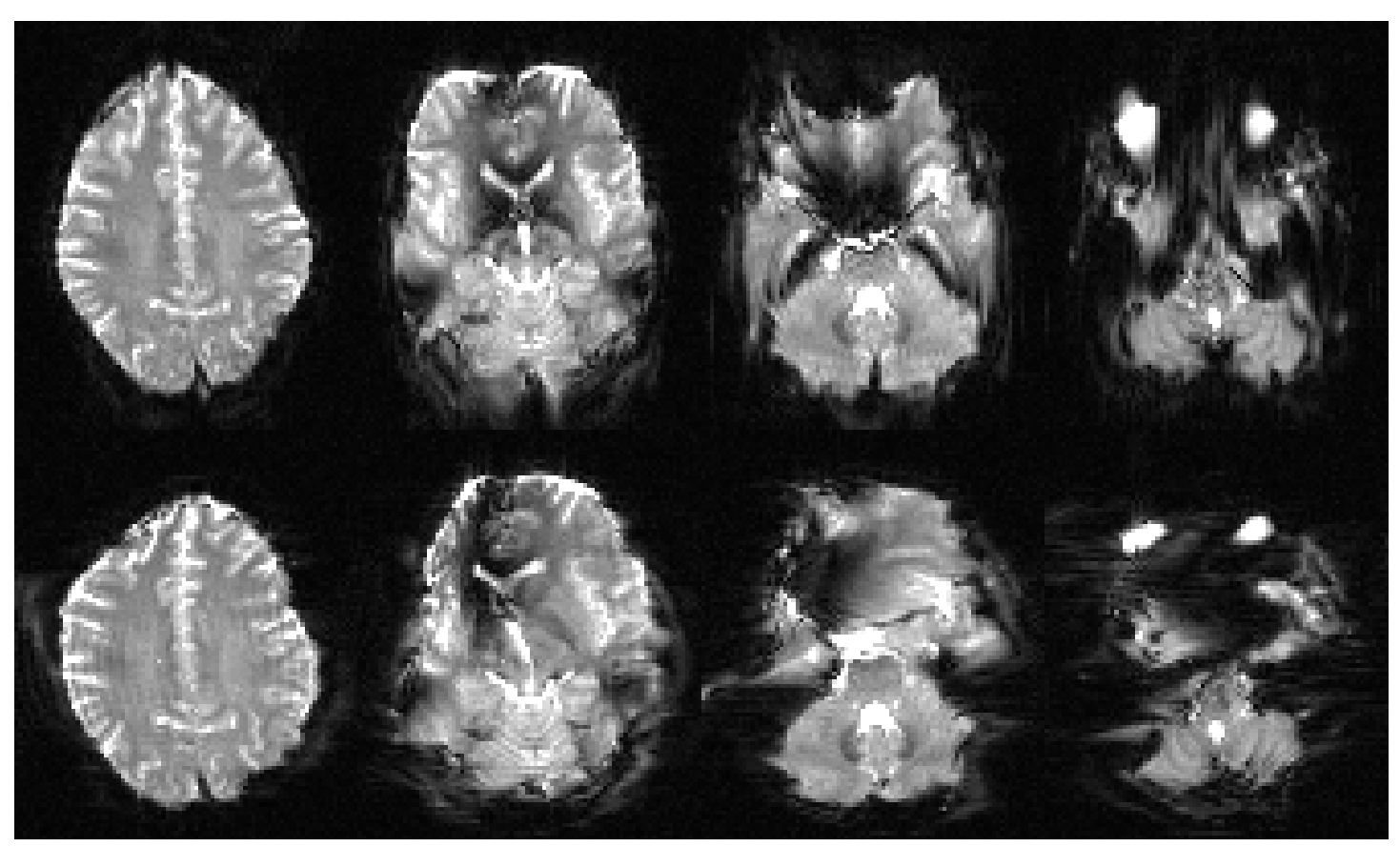

Fig. 1. Demonstration of EPI distortion. Selected EPI brain slices taken with two different phase-encoding gradient orientations: anteriorposterior for the upper row and left-right for the bottom row. The vertical, resp. horizontal deformation of the upper, resp. bottom row images is clearly visible.

obtained, e.g., from an image of a phantom. In the second step, this information is used to compensate for the deformation on real images. The major drawback of these methods is that it is impractical to build a phantom that would exactly duplicate the biological system being imaged [4], which limits the compensation only to field distortions other than those caused by the individual subject. Moreover, these other distortions are most likely already compensated for by the scanner manufacturer or operator. ${ }^{2}$

\section{Unwarping by registration}

We propose a third approach which, to the best of our knowledge, has never been applied to this particular problem. It consists of registering the distorted EPI image with a corresponding geometrically correct anatomical MRI image. In this way, we can recover the deformation $g$ from a single EPI slice obtained by an unaltered, standard procedure. The registration can be performed manually [8], but this is tedious, time consuming, and prone to errors. An automatic procedure is advantageous, because it is faster, more precise, and does not require an expert.

Our goal in this paper is to present an automatic registration algorithm we have developed specifically for this problem; i.e., identifying a non-linear unidirectional twodimensional warping. More precisely, given the observed EPI slice $f^{o}$ and the corresponding undistorted anatomical MRI reference slice $f^{r}$, the task is to find a warping $g$ so that the warped test image $f^{o}(g(x, y), y)$ matches as well as possible (in a sense defined later) the reference image $f^{r}$.

${ }^{2}$ This procedure is called shimming and is generally repeated before each series of acquisitions.

\section{Proposed Algorithm}

We categorize registration algorithms according to the warp space used. In general, a deformation function $g_{V}$ from a warp space $V$ is described by a finite set of parameters $c_{k}$ by means of a warping model.

At one end of the scale we have non-parametric, local methods. These methods are formulated either as variational, defining a scalar criterion to minimize, or (more generally) using PDEs. The continuously defined correspondence function that minimizes a given criterion (resp. that solves a given PDE) is sought for in a very large and unrestrictive function space, e.g., the Sobolev space $W_{2}^{2}$. The essence of these methods is entirely in the criterion (resp. PDE). The PDE come from the optical flow approach (gradient methods) [9], viscous fluid model [10-12], elastic deformations with physical analogs $[13,14]$ or without [15]. Some elastic deformations can also be modeled as potential fields [16].

At the other end, we have parametric, global methods that describe the correspondence function using a global model with a relatively small number of parameters [17]. The model mostly consists of expressing the warping function in a linear [3], global polynomial $[18,19]$, or harmonic basis [20]. For these methods, the deformation model corresponding to a specific warp space is as important as the criterion being minimized.

In this article, we consider mainly intensity-based registration methods, which directly take into account the voxel values, c.f. [21]. Other methods are based on matching surfaces [21-23], curves [24], or interpolating landmarks using radial basis functions, especially thin-plate splines $[21,25-$ 
which is compatible with the elasticity theory, as it corre-

\section{A. Semi-local model}

The model proposed in this article is situated between the above-mentioned local and global methods, combining the advantages of both.

We parametrize the warp space by a scale parameter $h$ and denote it $V_{h}$. The scale parameter corresponds loosely to the density of knots or landmarks. By changing $h$, we can approach either of the two limit cases or choose a compromise offering the best tradeoff. Big $h$ yields a global model which has just a few parameters. Such a model is rather constrained, which is approximatively equivalent to strong explicit regularization. On the other hand, a small $h$ gives a local model with many parameters, which generally leads to more complicated optimization. In exchange, a small $h$ generally permits one to approximate any given $g$ in $V_{h}$ well, because the space $V_{h}$ is big. (Arbitrarily small precision can be achieved as $h \rightarrow 0$.) This roughly corresponds to weak explicit regularization. Thus, the scale parameter can partly assume the role of an explicit regularization factor, unlike in local methods where the regularization is a part of the criterion.

In the next section, we give our motivation for the algorithm and for our particular choice of the warp modeluniform cubic splines represented by a linear combination of cubic B-splines $[28,29]$.

\section{B. Univariate case}

For the sake of explanation, let us begin with the onedimensional case; i.e., with the task of recovering an univariate warping function $g: \mathbb{R} \rightarrow \mathbb{R}$. An example of such a function could be the affine map $g(x)=\alpha x+\beta$. Such linear dependence is rather frequently encountered in practice; for example, it arises when the acquisition techniques use different coordinate systems or when there is a movement between acquisitions.

The univariate equivalent of our matching problem becomes $f^{o}(g(x)) \simeq f^{r}(x)$. The landmark method would consist of (either manually or automatically) identifying a set of landmark pairs $\left\{\left(x_{l}^{r}, x_{l}^{o}\right)\right\}_{l=1}^{L}$, so that a feature found at location $x_{l}^{r}$ in the reference image can be found (or associated with) a location $x_{l}^{o}$ in the observed test image. For interpolation, this gives a set of $L$ constraints for $g$ : $g\left(x_{l}^{r}\right)=x_{l}^{o}$. To get a well-posed problem, we shall require $g$ to minimize some criterion $J$. We do not want $J$ to penalize linear dependencies, i.e., we want $J$ to be zero for linear $g$. We want the resulting $g=\arg \min J$ to be linear with respect to the landmark coordinates $\left\{x_{l}^{o}\right\}$ and invariant with respect to a linear (affine) transformation of $\left\{x_{l}^{r}\right\}$, which means that if we take a linear combination of two sets of landmark coordinates, then the resulting $g$ should be the same linear combination of the solutions corresponding to the two sets of landmarks. In other words, we want a solution that is invariant to the choice of a particular coordinate system, or to the choice of units. The simplest criterion satisfying these requirements is $J=\int\left|g^{\prime \prime}(x)\right|^{2} \mathrm{~d} x$, sponds to 'strain' or 'bending' energy [25].

Besides interpolation, other approximation schemes can be applied, the most popular being least-squares fitting. It consists of minimizing an extended criterion $J^{\prime}=J+$ $\gamma \sum_{l}\left(g\left(x_{l}^{r}\right)-x_{l}^{o}\right)^{2}$. This has the advantage of accommodating uncertainty (noise) in landmark positions.

For both interpolation and least-squares fitting, as well as for any other criterion of the general form $J^{\prime}=J+$ $\sum_{l} \mathcal{L}\left(g\left(x_{l}^{r}\right), x_{l}^{o}\right)$ (with arbitrary function $\mathcal{L}$ ), the function $g$ that minimizes the criterion can be shown to be a cubic spline $[30,31]$; i.e., a piecewise cubic function that is twice continuously differentiable, with knots (the boundaries between polynomial pieces) at points $\left\{x_{l}^{r}\right\}$. In Appendix A, we show that the solution can be expressed as a linear combination of radial basis functions $\left|x-x_{l}\right|^{3}$. However, these functions are not convenient to work with because of their instability and global support. Fortunately, it is possible to localize the $\left|x-x_{l}\right|^{3}$ functions using divided differences, which yields a base made of cubic B-splines with local support that generates the same space [32].

The automatic landmark method is rather difficult to apply because there is no automatic landmark detection algorithm available that would be sufficiently robust and precise, especially for our class of medical images which typically exhibit only a few distinct features. Moreover, it is difficult to automatically find common features in both anatomical MRI and EPI modalities. For this reason, instead of trying to work with landmarks, we introduce a data criterion $E$ (defined in section II-D) taking into account the entire image and measuring the discrepancy between the warped version of the observed image $f^{\circ}(g(x))$ and the reference image $f^{r}(x)$. Then, we seek a deformation $g$ such that the two images are as similar as possible, i.e., when $E$ is minimized.

For the reasons mentioned above, we choose to search the warping $g$ also in the cubic spline space, like in the landmark case. However, there are now no explicit landmarks available to put the knots on. We also do not know, how much useful information each part of the image can provide. Therefore, we will distribute the knots uniformly over the image. It follows that the function $g$ will be a uniform cubic spline, which can be uniquely represented as a linear combination of uniformly-spaced cubic B-splines [28, 29]:

$$
g(x)=\sum_{k \in K} c_{k} \beta_{3}(x / h-k)
$$

where $K$ is the set of the indices of the spline functions, the support of which intersects with the image; $h$ is the knot spacing (the B-splines will be centered at points $k h$ for $k \in$ $K \subseteq \mathbb{Z}$ ). Working with uniform splines is also significantly faster with respect to non-uniform splines. See Appendix B for a definition of a cubic B-spline. Note that, in order to get a complete control over $g$, it is useful to put some spline knots 'outside' the image. For cubic splines, we need to put one such exterior knot at each side. Consequently, for an image size $N$ and knot spacing $h$, we have $N / h+2$ knots. 
We have thus transformed the registration task into a non-linear finite-dimensional optimization problem: Find a set of coefficients c minimizing some criterion $E$.

\section{Splines-a perfect fit}

Let us now show several important properties of the spline model.

(a) Good approximation properties - The error of a cubic spline approximation decreases asymptotically as $h^{4}$ (measured by any $L_{p}$ or $l_{p}$ norm, $p \in\{1,2, \ldots, \infty\}$ ). Quantitative analyses indicate that splines perform well in comparison with other wavelet-like basis functions [33].

(b) Speed-Cubic splines have a short compact support of length 4 . They are symmetric and piecewise cubic. To evaluate $\beta_{3}(x)$ at one particular point, only 5 arithmetic operations (additions or multiplications) and 3 comparisons are needed. In multiple dimensions, where we will use tensor products of cubic splines as basis functions (see Section II-E), the computational complexity stays low thanks to separability. The number of operations needed to evaluate $g$ also does not depend on the total number of basis functions (and thus the number of parameters $c_{k}$ ).

(c) Plausibility - The spline model corresponds to a wide range of physical situations where the restoring force can be approximated as being linearly dependent on the displacement. In such situations, the generated deformation is physically plausible. It is also a good approximation for cases when a better model is not known, such as the deformation of EPI images, even though it is not of mechanical origins.

(d) Simplicity - The model is linear in the parameters $c_{k}$ and polynomial with respect to the position $x$. It is thus possible to truncate the Taylor expansion such that it is exact in some neighborhood of $x$ with a typical diameter of $h / 2$.

(e) Scalability-Thanks to the $q$-scale relation $\beta_{3}(x / q)=$ $\sum_{k} \zeta(k) \beta_{3}(x-k)$, where $q \in \mathbb{N}$, we have the embedding $V_{h q} \subseteq V_{h}$; i.e., the transition from a coarse space $V_{h q}$ to a finer space $V_{h}$ is exact [34].

\section{Data criterion}

A reasonable and most often ([3]) used way to measure the discrepancy between two images is the sum of squared differences (SSD) criterion

$$
E=\sum_{i \in I}\left(f^{o}(g(i))-f^{r}(i)\right)^{2}
$$

where the sum is over all pixels in the image. Note that minimizing this criterion is equivalent to calculating the maximum likelihood estimate of the unknown parameters assuming that the difference is an i.i.d. Gaussian noise, and that the true test image is indeed a geometrically distorted version of the reference one. Moreover, the SSD criterion is also algorithmically advantageous because it is easy to evaluate (including its derivatives) and because it depends smoothly on the parameters. Interpolation (cf. Section II$\mathrm{G}$ ) is needed to evaluate this criterion, as it calls for values of $f^{o}$ on generally non-integer coordinates.

\section{E. Bivariate case}

The transition to the bivariate case is straightforward. The criterion becomes

$$
E=\sum_{\mathbf{i} \in I}\left(f^{o}(\mathbf{g}(\mathbf{i}))-f^{r}(\mathbf{i})\right)^{2}
$$

where we have taken the convenience notation

$$
\mathbf{g}(\mathbf{i})=\mathbf{g}(i, j)=\left[\begin{array}{c}
g(i, j) \\
j
\end{array}\right]
$$

and where the warping function is now described by a twodimensional array of coefficients

$$
\begin{aligned}
& g(\mathbf{x})=g(x, y)=\sum_{\mathbf{k} \in K} c_{\mathbf{k}} \boldsymbol{\beta}_{3}(\mathbf{x} / \mathbf{h}-\mathbf{k})= \\
& =\sum_{\left(k_{x}, k_{y}\right) \in K} c_{k_{x}, k_{y}} \beta_{3}\left(x / h_{x}-k_{x}\right) \beta_{3}\left(y / h_{y}-k_{y}\right)
\end{aligned}
$$

Tensor products of splines were also used in [35].

\section{F. Optimization algorithm}

The criterion is minimized with respect to the coefficients $c_{k}$ using a regularized version of the Newton method [3, 36], inspired by the Marquardt-Levenberg algorithm. This algorithm smoothly varies between the gradient-descent and the Newton approach, which gives it robustness and quadratic convergence near the optimum.

The algorithm uses first two derivatives of the criterion $E$ with respect to $c_{k}$; i.e., $\nabla_{c} E$ and $\nabla_{c}^{2} E$. Thanks to the spline representation (2), the derivatives can be calculated exactly and at a small cost. As the number of components each pixel contributes to remains constant, the cost of evaluating $E, \nabla_{c} E$ and $\nabla_{c}^{2} E$ does not depend on the number of coefficients $c_{k}$ (or, equivalently, the spacings $h_{x}, h_{y}$ ) used to describe the deformation. See Appendix C for explicit formulas.

At each step we update the vector of all coefficients $\mathbf{c}$ to $\mathbf{c}+\Delta \mathbf{c}$ by taking

$$
-\left(\nabla_{c}^{2} E(\mathbf{c})+\lambda \mathbf{I}\right) \Delta \mathbf{c}=\nabla_{c} E(\mathbf{c})
$$

where the regularization factor $\lambda$ is divided by a constant $\lambda_{f}$ if the previous step resulted in a decrease in $E$, otherwise it is multiplied by the same amount. ${ }^{3}$ We iterate as long as the relative and absolute change of $E$ stay above a priori given thresholds.

When the number of coefficients exceeds a certain limit, the Hessian matrix $\nabla_{c}^{2} E$ gets too big for the linear equation set $(7)$ to be efficiently solvable and this algorithm ceases to be practicable. Note that the theoretical computational complexity of solving (7) increases as $h^{-6}$. The asymptotical memory requirements grow as $h^{-4}$. While iterative

\footnotetext{
${ }^{3}$ We use $\lambda_{f}=10$.
} 
linear equation solvers generally speed up the solution of $(7)$, it is at the cost of a loss in precision, and the overall gain in our case is insignificant.

When the size of the Hessian matrix exceeds our computational capacities, we replace the Marquardt-Levenberg optimizer by a conjugate gradient method [36], which converges quadratically too without explicitly calculating and storing the Hessian matrix. Even though the conjugate gradient method needs more iterations for smaller problems, it outperforms Marquard-Levenberg for bigger ones.

\section{G. Image interpolation model}

To calculate the derivatives, as well as to evaluate the criterion (4), an image interpolation model is needed to get a continuous form ${ }^{c} f(\mathbf{x})$ from a discrete image $f^{o}(\mathbf{i})$. Because of their good approximation properties, simple analytic form and effective algorithms available, we use cubic B-splines here as well

$$
\begin{aligned}
& { }^{c} f(x, j)=\sum_{i} b_{i j} \beta_{3}(x-i) \\
& \text { where } \quad{ }^{c} f(i, j)=f^{o}(i, j), \forall(i, j) \in I
\end{aligned}
$$

The coefficients $b_{i j}$ can be obtained prior to registration by an efficient filtering algorithm [29], which incurs negligible overhead. For the filtering, we are using mirror boundary conditions on the image; in this way we have the same number of coefficients $b_{i j}$ as there are pixels in the original image.

\section{H. Multiresolution}

The robustness and efficiency of the algorithm can be significantly improved by a multiresolution approach: The task at hand is first solved at a coarse scale. Then, the results are propagated to the next finer level and used as a starting guess for solving the task at that level. This procedure is iterated until the finest level is reached.

In our algorithm, multiresolution is used twice. First, we build an image pyramid: a set of gradually reduced versions of the original image [37]. This pyramid is compatible with our image representation (8) and is optimal in the $l_{2}$-sense (i.e., in the sense of the criterion (4)), which ensures that the approximation made by substituting the lower resolution image is the best possible. Based on an image of size $N_{x} \times N_{y}$, we create a sequence of images with sizes $\mathcal{M}_{I}=\left\{\left(N_{x}^{1}, N_{y}^{1}\right),\left(N_{x}^{2}, N_{y}^{2}\right), \ldots,\left(N_{x}^{m_{I}}, N_{y}^{m_{I}}\right)\right\}$, where $N_{x}^{m_{I}}=N_{x}, N_{y}^{m_{I}}=N_{y}$, and where $N_{x}^{j}=\left\lfloor N_{x}^{j+1} / 2\right\rfloor$, $N_{y}^{j}=\left\lfloor N_{y}^{j+1} / 2\right\rfloor$. The optimum starting size $\left(N_{x}^{1}, N_{y}^{1}\right)$ depends on the image. We chose $16<\min \left(N_{x}^{1}, N_{y}^{1}\right) \leq 32$, which works well in most cases.

Second, we use multiresolution also for the warping function. We start with a deformation $g$ described with very few parameters $c_{k}$, and with a large distance $h$ between knots. After the optimization of $c_{k}$ is complete, we halve the distance between knots. This approximately corresponds to doubling the number of knots in each direction, i.e., quadrupling the number of coefficients $c_{k}$. Because of the two-scale spline relation, we can exactly represent the warping function from the old, coarse space, in the new, finer space. More precisely, the sequence of knot spacings is going to be $\mathcal{M}_{W}=\left\{\left(h_{x}^{1}, h_{y}^{1}\right),\left(h_{x}^{2}, h_{y}^{2}\right), \ldots,\left(h_{x}^{m_{W}}, h_{y}^{m_{W}}\right)\right\}$, where $h_{x}^{1}=N_{x}, h_{y}^{1}=N_{y}$; the final element $\left(h_{x}^{m_{W}}, h_{y}^{m_{W}}\right)$ corresponds to the user-chosen target grid size. The sequence obeys $h_{x}^{j+1}=h_{x}^{j} / 2, h_{y}^{j+1}=h_{y}^{j} / 2$. The process starts with $g$ being identity.

The global strategy that combines the two multiresolutions is depicted in Figure 2. Symbolically, to construct the double multiresolution $\mathcal{M}$ we first extend the shorter of $\mathcal{M}_{I}, \mathcal{M}_{W}$ to the length $m=\max \left(m_{I}, m_{W}\right)$ by repeating the last element. Then, $\mathcal{M}$ consists of pairs of elements from $\mathcal{M}_{I}, \mathcal{M}_{W}$ in alternate progression: $\mathcal{M}=\left\{\left(\mathcal{M}_{I}^{1}, \mathcal{M}_{W}^{1}\right),\left(\mathcal{M}_{I}^{1}, \mathcal{M}_{W}^{2}\right),\left(\mathcal{M}_{I}^{2}, \mathcal{M}_{W}^{2}\right),\left(\mathcal{M}_{I}^{2}, \mathcal{M}_{W}^{3}\right)\right.$, $\left.\ldots,\left(\mathcal{M}_{I}^{m}, \mathcal{M}_{W}^{m}\right)\right\}$.

The consequence of using multiresolution is that the algorithm works best for images and deformations that follow the multiresolution model; i.e., when a low resolution version is a good approximation of the finer resolution version.

\section{Invertibility}

We implicitly assumed that the deformation $\mathbf{g}$ is recoverable and recoverability requires invertibility. In case the EPI artifacts cause the signal from adjacent pixels to blend due to the fold-over effect, the invertibility condition might not be satisfied and the deformation is not recoverable. However, this does not happen in practice. To ensure the stability of the registration, it is necessary to enforce the invertibility also on the trial solutions during the optimization process, i.e., to perform constrained optimization. If we assume that the deformation conserves orientation, the sufficient condition ensuring local invertibility is the positivity of the Jacobian, $\operatorname{det}\left(\nabla_{\mathbf{x}} \mathbf{g}\right)>0$, everywhere. In our case, the invertibility condition reduces to $\forall(x, y) \in S ; \partial g / \partial x>0$, where $S$ is the image domain.

Although an iterative algorithm can be found verifying this condition exactly, it is not practical to apply it for performance reasons. Instead of checking that $\partial g / \partial x>$ 0 on a continuous domain, we sample this condition on pixel coordinates. At this scale, the two conditions are essentially equivalent.

There is little hope of finding an algorithm capable of solving a constrained optimization problem of our complexity (highly non-linear criterion, hundreds of parameters and tens of thousands of constraints) in a reasonable time. We have therefore chosen to convert the constrained search into an unconstrained one using an exponential penalty cost function. A set of constraints

$$
0<u_{\mathbf{p}}=\left.\frac{\partial g}{\partial x}\right|_{(x, y)=\mathbf{p}}
$$

is replaced by a penalty function $E_{p}$

$$
E_{p}=\gamma \sum_{\mathbf{p}} \mathrm{e}^{-\alpha u_{\mathbf{p}}}
$$




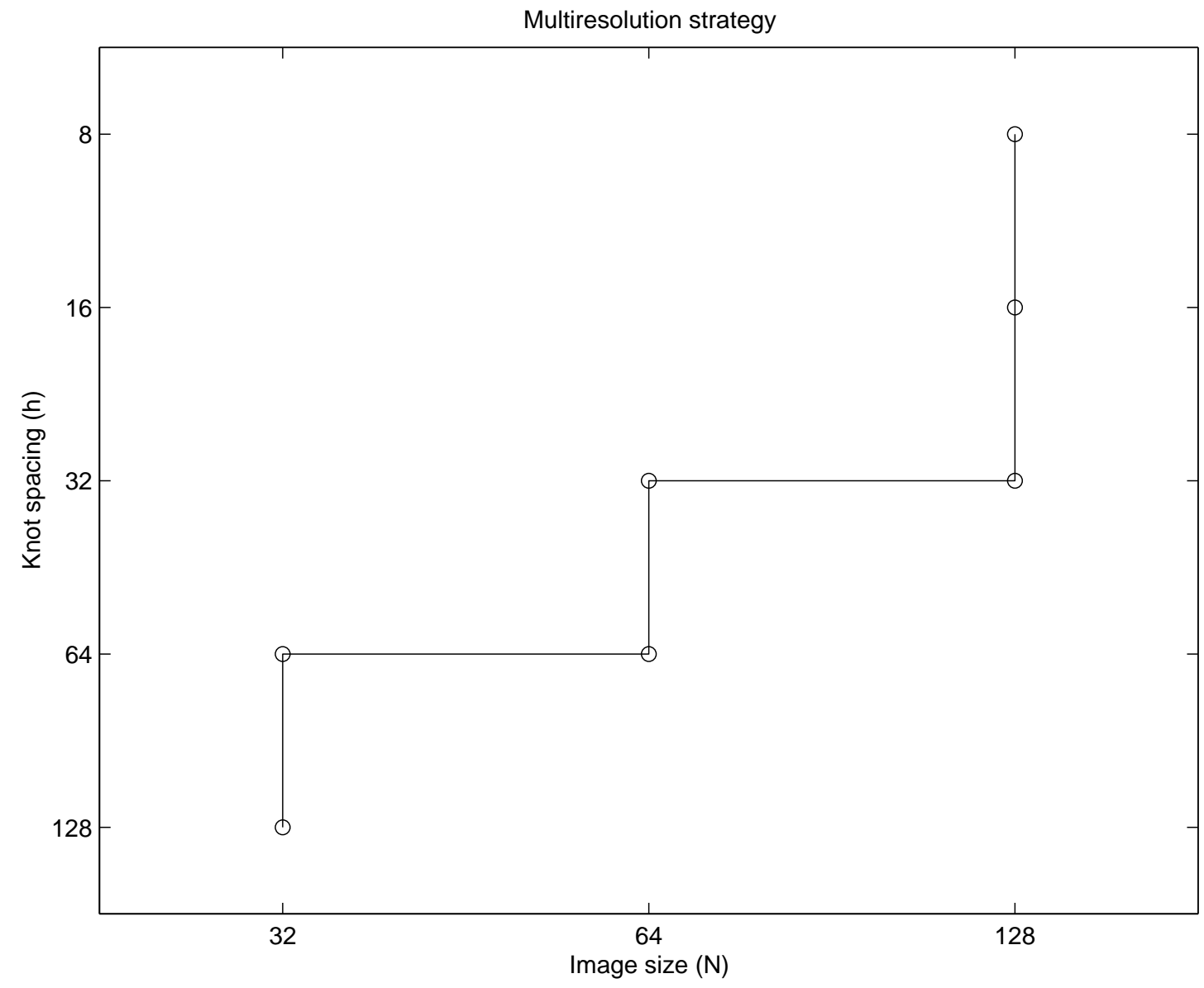

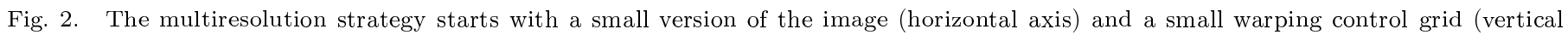
axis). After several steps, and by augmenting resolution alternatively in the two domains, we reach the original image resolution and the desired size of the control grid.

Consequently, we will minimize the combined criterion

$$
E_{c}=E+E_{p}
$$

The choice of the constants $\alpha$ and $\gamma$ is a tradeoff between speed and precision. As $\alpha$ increases, the penalty function gets steeper, which improves precision in the vicinity of the constraints. At the same time, the criterion becomes highly non-linear, which slows down the optimization. We set $\alpha$ and $\gamma$ so that $E_{p} \approx E$ on the boundary of the permissible space and $E_{p} \approx 10^{-5} E$ for the initial configuration, when $g$ is an identity.

\section{J. Preprocessing}

To apply the SSD criterion, we need to make the test and reference images more similar, so that their difference after warping is as close as possible to white noise. We choose therefore to apply a preprocessing step that consists of high-pass filtering and histogram equalization. The effect can be seen in Figure 3.

The preprocessing makes it unnecessary to add a special parameter accounting for differences in intensity profiles of the two images. It also helps to compensate for the intensity distortion due to in-plane dephasing, which is also caused by the magnetic field inhomogeneity. The dephasing cannot be compensated completely, as it is impossible to differentiate between effects of dephasing and warping on a single pixel.

\section{EXPERIMENTS}

\section{A. General comments}

We tested the performance of our algorithm on several hundreds of images. In addition, we compare to the automatic method the results of the registration of thirty image pairs by three different people, including one experienced practitioner. For the manual registration, we use the standard thin-plate spline method $[8,25,38]$.

Unless stated otherwise, the tests are performed on $128 \times 128$ pixel spin-echo anatomical MRI images of the brain, on a SUN Ultra workstation, and the published numbers are arithmetical means of the results of experiments made on all of the 30 horizontal slices of a brain volume. A typical pair consisting of corresponding anatomical and EPI images is shown in the upper row of Figure 3. 

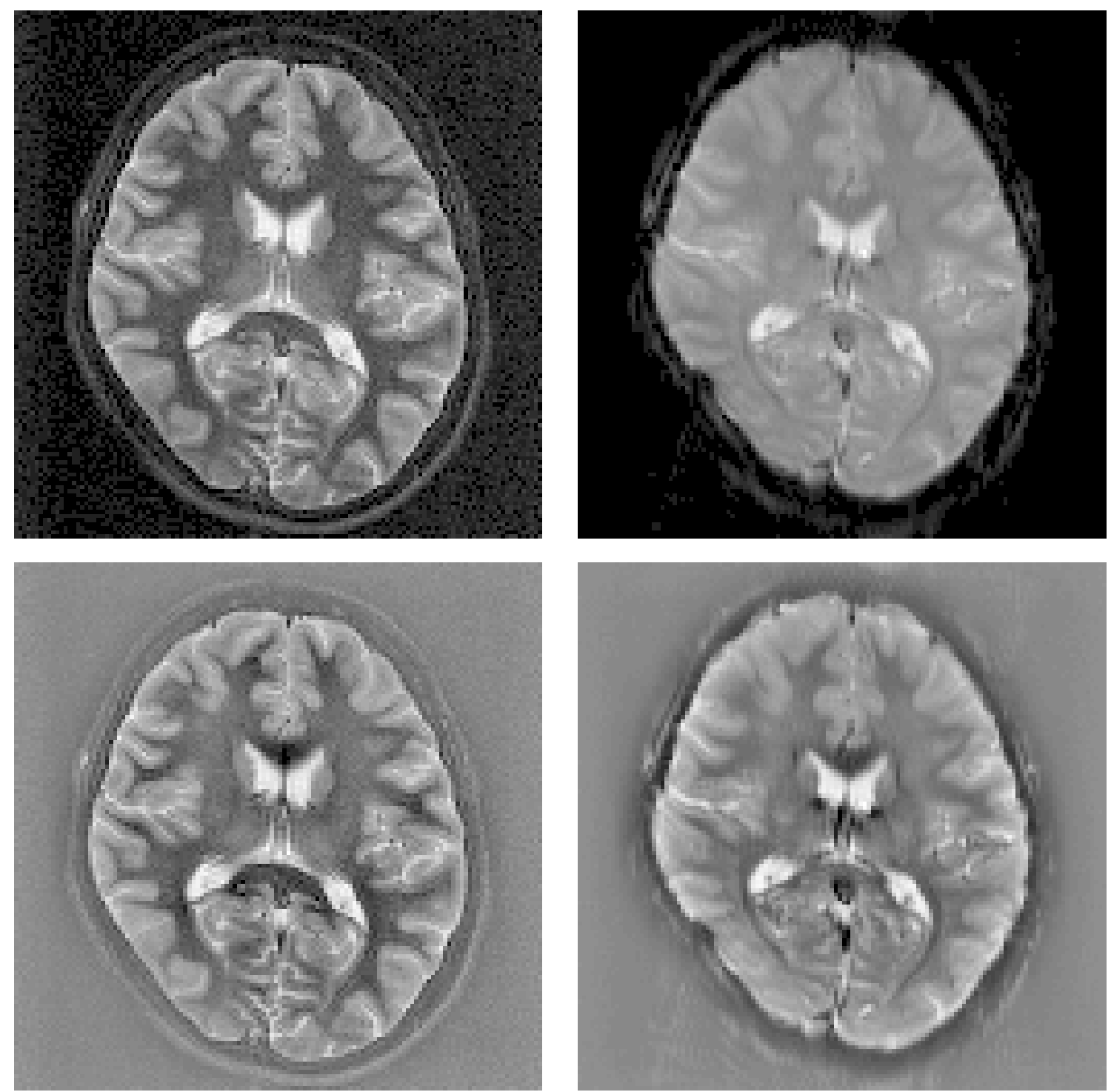

Fig. 3. An anatomical MRI image (top left) and a corresponding EPI image (top right). Both images represent a horizontal slice through the middle of a human brain. The bottom line shows the same images after preprocessing.

\section{B. Error measurement}

As a main measure to compare different solutions to the registration problem, we use the warping index defined in [3] as $\varpi=\frac{1}{\|R\|} \sum_{\mathbf{i} \in R}\left|g(\mathbf{i})-g^{*}(\mathbf{i})\right|$, where $g^{*}$ is the reference solution (ideally, the true deformation), and $R \subseteq I$ is the region of interest (in our case, the interior of the brain). This corresponds to the mean per-pixel precision of the result.

We shall also use the sum of squared differences (SSD), as defined by (3). It measures the similarity of the reference 2 . and warped test images as perceived by the algorithm, and corresponds to the quantity minimized.

\section{Sources of error}

There are several reasons why we cannot expect a perfect registration (indicated by $\varpi=0$ ).

1. Different images — Despite the assumptions made when de- riving our criterion, the images we are asked to register are not geometrically deformed versions of each other. To this we must add the effects of discretization, quantization, and noise. Any of them may result in spurious minima of the criterion $E$, misleading the algorithm. This problem can be alleviated by preprocessing, or by choosing a different criterion, but it can never be completely removed. The situation is more favorable when a small number of parameters is sought, for the random effects tend to cancel out.

Local minima - The algorithm with its local vision cannot distinguish between local and global minima of the criterion and can therefore get trapped in a local minimum. Multiresolution improves significantly this aspect, but it does not solve it entirely. Again, the situation is much more favorable for a small number of parameters.

3. Warp space-The true warping does not necessarily belong to the warp space $V_{h}$. We define $\varpi_{\min }$ as the min- 
imum achievable registration error, which is equal to the distance between the true warping and the closest point in the warp space $V$. We call this closest point (which is the best possible approximation of $g$ in $V$ ) a projection $\mathcal{P}_{V} g$. In the $L_{2}$ or $l_{2}$ sense, it is equal to $\sum_{k} \mu_{k} \varphi_{k}$, where the coefficients $\mu_{k}$ verify $\left\langle g, \varphi_{l}\right\rangle=\sum_{k} \mu_{k}\left\langle\varphi_{k}, \varphi_{l}\right\rangle$, and where we have denoted the basis functions by $\varphi_{k}$ in order to simplify the notation. For other norms, iterative procedure must be used. In a controlled experimental environment, we can thus calculate $\varpi_{\min }$ exactly and compare it with the results of our algorithm.

4. Lack of details - The precision is limited by the local resolution of the images. When two corresponding sharp edges occur in both images, sub-pixel registration precision is often attainable. On the other hand, in homogeneous, texture-less regions, there is little hope of recovering any information whatsoever.

5. Criterion surface complexity-Depending on the images being registered, the dependence of the criterion on the parameters can be non-linear, non-convex, and generally very complicated. In such a situation, the convergence is extremely slow. If the time is limited, we must stop the algorithm before any significant improvement has been realized.

6. Numerical precision-Insufficient numerical precision can hinder the performance of the optimizer. We have encountered this problem only rarely, when iterative methods were used to solve the normal equation set (7).

We have tried to design the experiments to separate the effect of the above-mentioned factors whenever possible; however, this will rarely be the case in a real-life situation.

\section{Deformation generator}

To test our algorithm, we have implemented a waveletbased deformation generator. We want to generate a random Sobolev-type deformation - a deformation lying in a prescribed Sobolev space $W_{2}^{r}$. The parameter $r$ refers to the regularity. It is equal to the number of derivatives in the $L_{2}$-sense and it is also strongly related to point-wise differentiability. The higher the $r$, the more regular are the functions from $W_{2}^{r}$. Wavelets are known to be good bases for functions lying in Sobolev spaces and the decay of wavelet coefficients across scales is directly related to a Sobolev-type regularity. Let $\theta_{j, k}$ denote the coefficients of a wavelet expansion ${ }^{4}$

$$
g(x)-g_{I}(x)=\sum_{j, k} \theta_{j, k} \psi\left(2^{j} x-k\right)
$$

where $g_{I}$ is an identity transform and $\psi$ is an orthogonal wavelet. Then the displacement $g-g_{I}$ belongs to a Sobolev space $W_{2}^{r}$ if and only if

$$
\sum_{j, k} 2^{2 j r}\left|\theta_{j, k}\right|^{2}<\infty
$$

\footnotetext{
${ }^{4}$ For brevity, we shall deal here with the unidimensional case only.
}

provided that the regularity of $\psi$ is greater than $r$ [39]. It follows that, for (14) to hold, the necessary condition is

$$
\exists C \in \mathbb{R} ; \forall j, k ;\left|\theta_{j, k}\right|^{2}<C \xi^{j} \quad \text { with } \quad \xi=2^{-2 r}
$$

Practically, we generate our deformations using zeromean, normally distributed coefficients with variance

$$
\mathbf{E}\left\{\theta_{j, k}^{2}\right\}=\sigma_{0}^{2} \xi^{j}
$$

where $\sigma_{0}^{2}$ governs the total energy of the deformation. Such a deformation will be denoted $W_{H}\left(\sigma_{0}, r\right)$, where the relation between $r$ and $\xi$ is given by (15). Note that the generated displacements are white noise for $r=0$, and become progressively smoother as $r$ increases. The regularity of the deformation converges to that of the generating wavelet $\psi$ for $r \rightarrow \infty$. For moderate to large $r$, we get a hierarchical warping: a deformation comprising displacements at several scale levels with gradually decreasing amplitudes, from important large-level deformation towards progressively smaller finer-level details. The algorithm should work well for such deformations which are compatible with the multiresolution strategy.

Finally, the deformation can be projected onto $V_{h}$ if needed, in which case we denote it $W_{H}\left(\sigma_{0}, r, h\right)$. Typically, we use Battle-Lemarié wavelets of order $4, \sigma_{0}=5$ and $r=0.5 \sim 1.6$, depending of what aspect of the algorithm we want to highlight. For some experiments, we have also added an affine component. Examples of generated deformations are shown in Figure 4.

\section{E. Ideal case}

We begin our series of experiments with an ideal case: the test $\left(f^{o}\right)$ and reference $\left(f^{r}\right)$ images are identical except for a known transformation, no noise is present. We use a random Sobolev deformation $W_{H}(5,1.6,8)$ as defined in the previous section. We show how the criterion $E$ and the warping index $\varpi$ decrease with the knot spacing $h$. Moreover, we want to demonstrate the advantage of using cubic splines to represent the warping, as opposed to linear and quadratic ones ${ }^{5}$ (Linear splines are sometimes used for motion estimation $[40,41]$.)

Figure 5 displays $E$ and $\varpi$ as a function of the degree $n$ of the splines used to represent the deformation $g$, and the knot spacing $h$. It clearly shows the benefit of using a high degree $n$ in high-precision applications. The minimum achievable error $\varpi_{\min }$ (cf. Section II-Cb) is shown by a dotted line for the cubic case and marked optimal.

\section{F. Image interpolation order}

As many other registration algorithm use only linear interpolation on the image, we want to show in this experiment that higher-order image interpolation is advantageous from the point of view of both precision and speed.

Table I demonstrates the dependency of the registration accuracy and speed on the degree $n_{i}$ of the splines used

${ }^{5}$ For splines of even degrees, there is an additional shift term of $1 / 2$ in (2), e.g., $\beta_{2}(x / h-k+1 / 2)$, which minimizes the number of knots needed. Other changes are straightforward. 

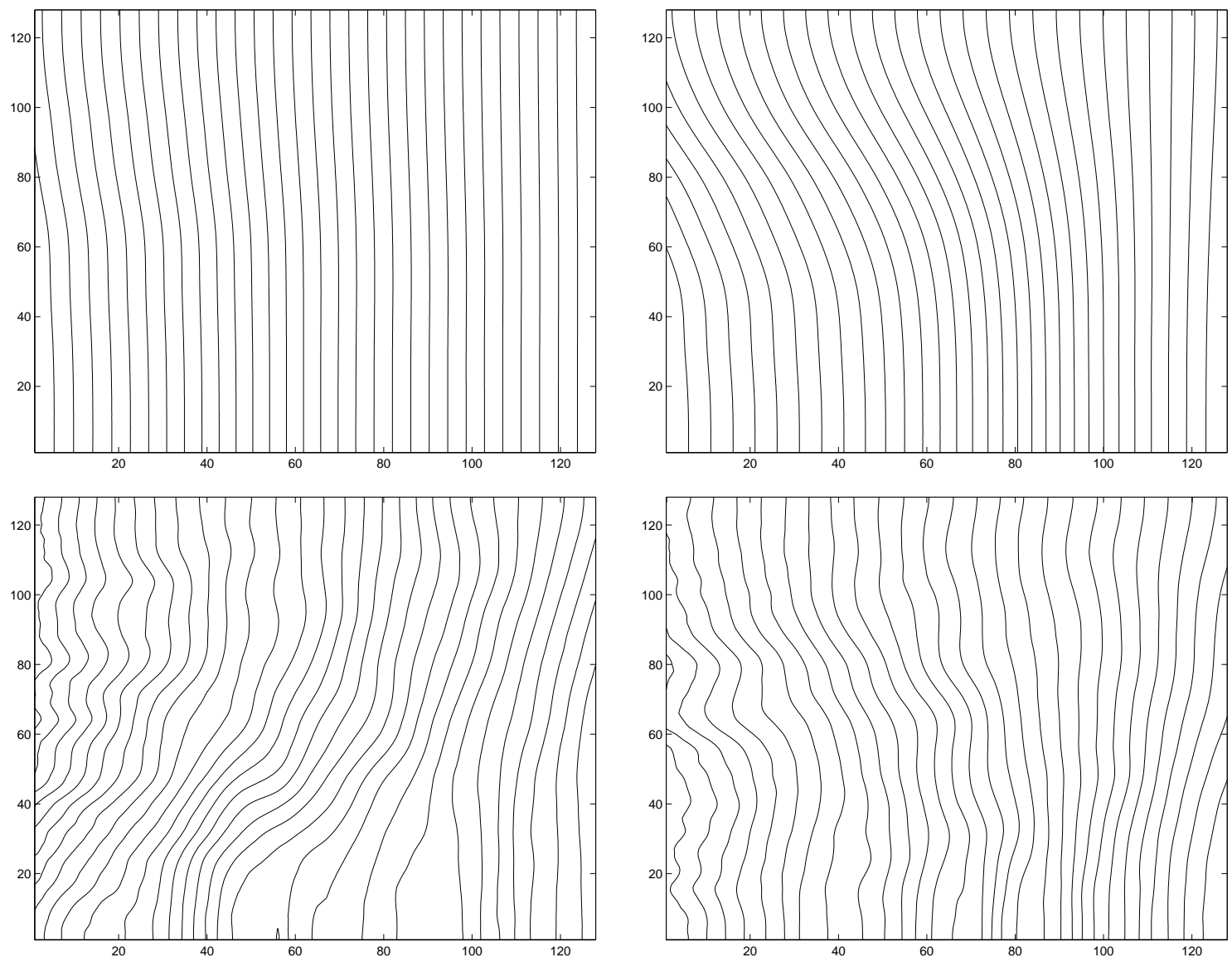

Fig. 4. Examples of deformation presented as contour plots. The top row ones were generated with $W_{H}(10,1.6)$, the bottom ones with $W_{H}(10,0.6)$.
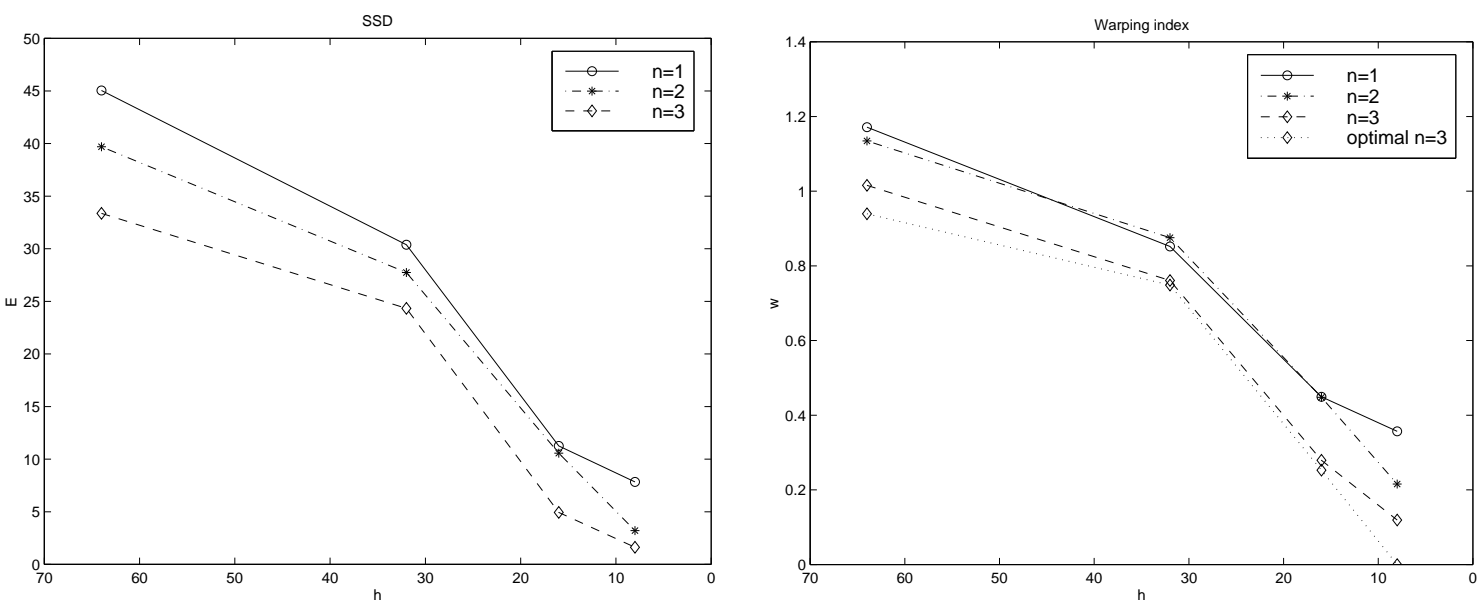

Fig. 5. The quality of the registration as a function of the warp spline degree and the knot spacing. The initial values ranges (prior to the registration) were $E=150$ and $\varpi=3.5$. Each point shown is an average of thirty experiments. 
for image interpolation. As a deformation, we have chosen $W_{H}(5,0.5,64)$. The stopping criterion was identical in all cases.

Besides providing a less accurate approximation, linear splines are penalized by not providing a meaningful second derivative estimate of the image, which forces us to use the slower conjugated gradient algorithm in place of the Marquardt-Levenberg optimizer.

The table shows that, although all results are accurate to a fraction of a pixel, the convergence speed varied a lot. This proves that the benefit from better approximation properties of higher-order splines with respect to linear, or even quadratic ones, indeed overweights the increase in computational complexity per iteration. This is consistent with other findings in the literature [3]. Being able to better estimate the image derivatives $\nabla_{\mathbf{x}}{ }^{c} f$, one is able to better estimate the criterion derivatives $\nabla_{c} E$ and $\nabla_{c}^{2} E$. This permits the optimization algorithm to acquire a more precise local model of $E(\mathbf{c})$, which in turn leads to more efficient optimization steps and faster convergence. The ability to precisely represent an image from a few samples is crucial at coarse levels of the image pyramid. The iterations there are relatively inexpensive compared to finer levels because we process much less data; it is therefore beneficial to get as close as possible to the optimum. In this manner we provide a good starting estimate for the next finer level, where each iteration costs at least 4 times more, and thus saving the overall effort.

\section{G. Noisy case}

Figure 6 demonstrates the dependence of the registration accuracy on the signal-to-noise ratio. For this series of experiments, the test images were obtained from a known transformation of a reference image with a various level of white Gaussian noise added. Here, an in-space deformation $W_{H}(5,1.16,32)$ was used. We observe that the degradation of the algorithm's performance is graceful for SNR $>10 \mathrm{~dB}$.

\section{H. Out-of-space deformation}

This experiment illustrates the behavior of the algorithm as the deformation progressively moves out of the warp space. Specifically, we have used $W_{H}(5,0.5,32)+$ $\alpha W_{H}^{\prime}(5,0.5)$ with $0 \leq \alpha \leq 1$, where the first term corresponds to the in-space part of the deformation and $W_{H}^{\prime}$ is the displacement associated with $W_{H}$, i.e., $W_{H}-g_{I}$. Figure 7 compares the attained and theoretically attainable warping index and the corresponding SSD.

It can be seen that, the experimentally recovered deformation is almost as close to the true one as theoretically possible, with the exception of $\alpha=0$; i.e., a deformation lying exactly in the warp space. On the other hand, we obtain mostly a smaller SSD than what corresponds to the projection. This demonstrates that the relationship between the criterion we optimize $(E)$ and the true error we make $(\varpi)$ is far from straightforward: the minimum of the difference $(E)$ does not correspond exactly to the true solution as measured by $\varpi$. We will see this sort of behavior in Section III-J, too.
TABLE III

Manual Versus automatic Registration. $\varpi$ is the WARPing INDEX, $E$ IS THE MEAN-SQUARE DIFFERENCE.

\begin{tabular}{|c||r|r|r|r|r|}
\hline & before & manual & automatic & ideal & true \\
\hline \hline$\varpi$ & 2.60 & 1.01 & 0.44 & 0.19 & 0 \\
\hline$E$ & 246 & 178 & 161 & 163 & 162 \\
\hline time & & $\sim 5 \mathrm{~min}$ & $\sim 30 \mathrm{~s}$ & & \\
\hline
\end{tabular}

\section{Multiresolution strategy}

In Table II, we compare three multiresolution strategies: the strategy actually used, a strategy without multiresolution in the image size, and a strategy without multiresolution in the warping grid size. The results show that both multiresolutions improve accuracy as well as speed of the algorithm because they reduce the amount of data to be treated and provide a smoothed version of the problem.

Multiresolution in the warp grid size significantly improves accuracy because it avoids that the algorithm is trapped in a local minimum. The multiresolution in the image size reduces the amount of data to be treated and consequently also the execution time, mainly for coarse control grids.

The joint strategy combines the advantages of both multiresolutions and yields the best results.

\section{J. Artificial EPI images}

By filtering and performing histogram modification of the anatomical MRI images, we obtain images that are visually equivalent to the corresponding EPI images (compare Figures 8 and 3 ) and we use them as test images. We warp the anatomical images with a known deformation $W_{H}(5,0.9)$ and use them as reference images.

The reference/test image pairs are registered automatically as well as manually using the landmark method by 3 people, including one experienced practitioner. The results are presented in Table III. For the manual case, the best results obtained (in the sense of $\varpi$ among all the attempts of all the participants) are shown. The column marked ideal shows the minimum attainable warping index $\varpi_{\text {min }}$, given the fact that the target deformation does not belong to the space searched by the algorithm. The results demonstrate that the automatic method is vastly superior to the manual one.

\section{K. Real EPI images}

Figure 9 shows a typical pair of corresponding anatomical and EPI images with superimposed contours of the anatomical image before and after manual and automatic registration. It illustrates that the automatic procedure leads to subjectively comparable or better results than the manual one.

\section{Conclusion}

We have suggested a new approach for undoing nonlinear unidirectional deformations in EPI images. We pro- 
TABLE I

The quality of the registration as a function of the image interpolation spline degree. The warping function was INTERPOLATED BY CUBiC SPLINES.

\begin{tabular}{|c||r|r|r|r|r|r|r|}
\hline \multicolumn{1}{|c||}{$h$} & & \multicolumn{3}{|c|}{32} & \multicolumn{3}{|c|}{16} \\
\hline$n_{i}$ & before & 1 & 2 & 3 & 1 & 2 & 3 \\
\hline \hline$E$ & 113.4 & 2.53 & 1.84 & 1.50 & 1.12 & 1.00 & 0.58 \\
\hline$\varpi$ & 2.93 & 0.130 & 0.111 & 0.098 & 0.074 & 0.067 & 0.057 \\
\hline time $[\mathrm{s}]$ & & 1010 & 766 & 69 & 1983 & 1500 & 103 \\
\hline
\end{tabular}

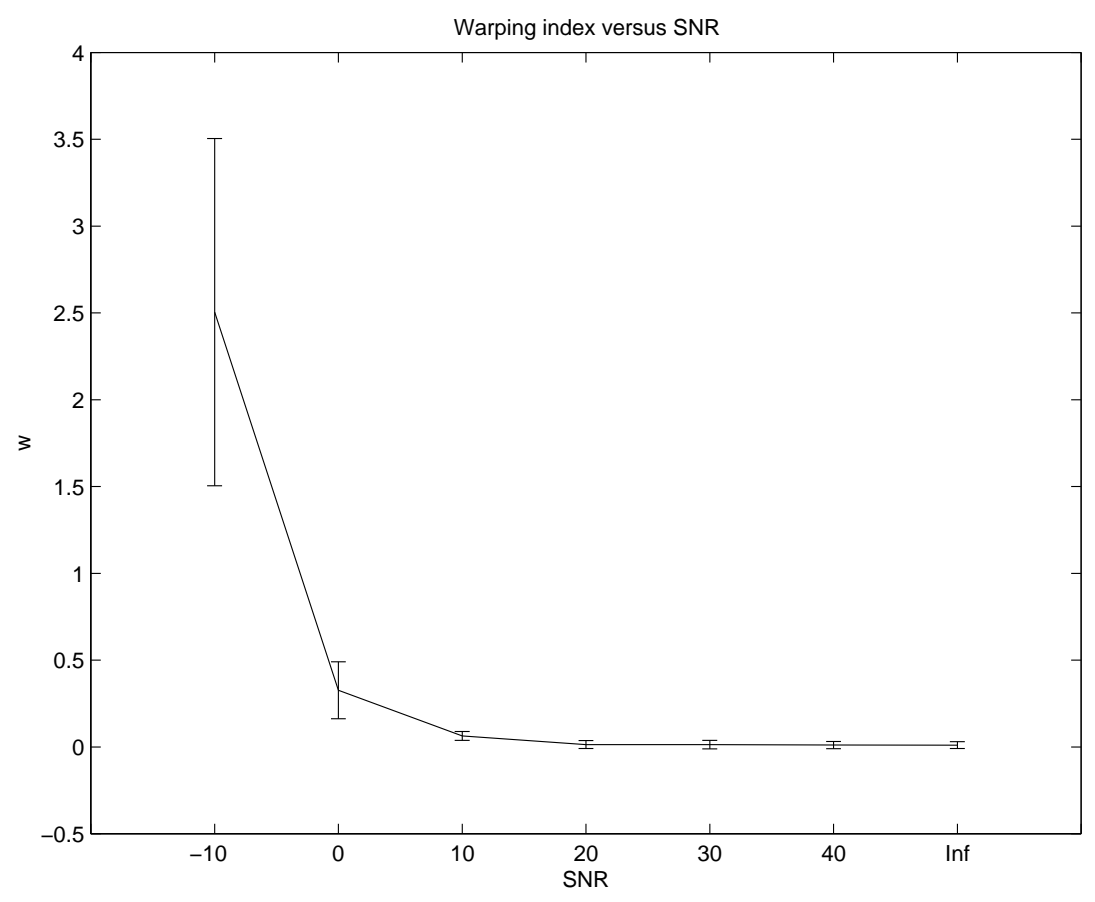

Fig. 6. The quality of the registration as a function of the SNR in dB. $h=32, \varpi_{\text {before }}=3.34$. The error bars mark one standard deviation.
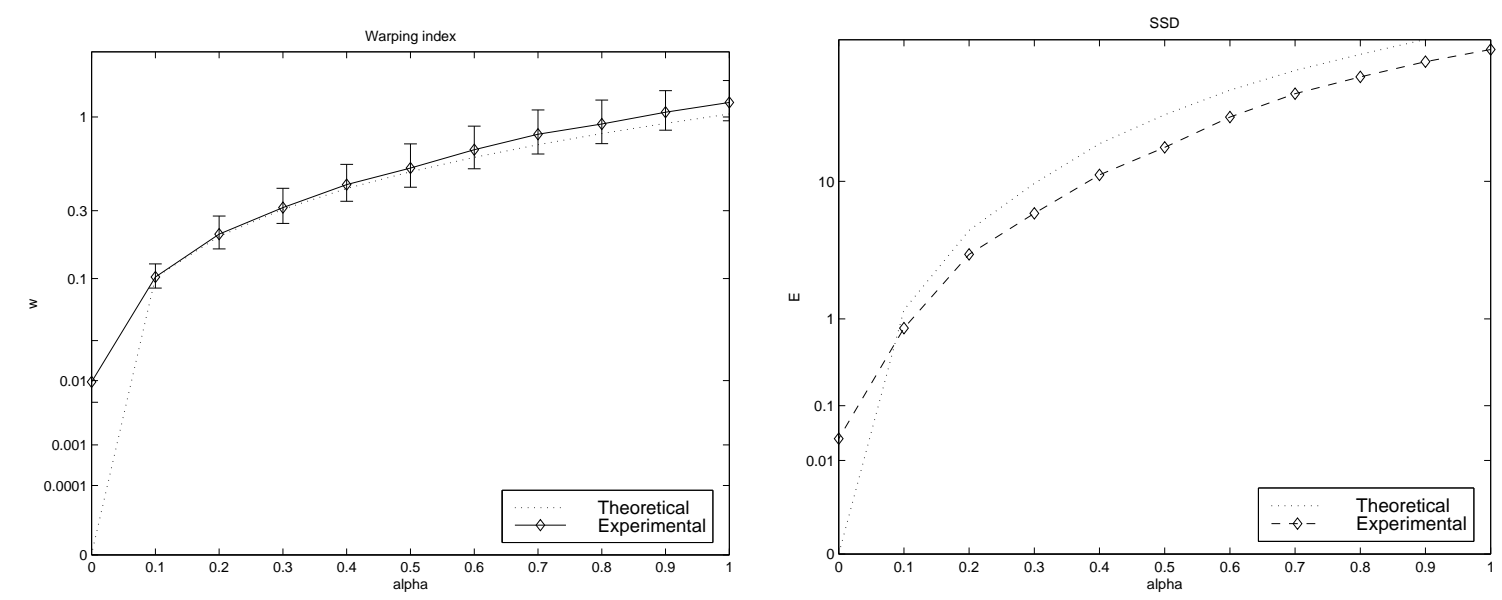

Fig. 7. Warping index (left) and SSD (right) for an out-of-space deformation. The mean initial values (prior to the registration) ranged between $E=146, \varpi=2.8$ for $\alpha=0$ and $E=181, \varpi=3.47$ for $\alpha=1$. Error bars show one standard deviation. 
Different multiresolution strategies. Image size is Denoted by $N$, Grid SpaCing By $h$. From top to bottom: joint, image Size ONLY, AND WARP GRID SIZE ONLY MULTIRESOLUTIONS.

Warping index before registration $\varpi=9.3$.

\begin{tabular}{|c|c|c|c|c|c|c|c|}
\hline$h$ & 128 & 64 & 64 & 32 & 32 & 16 & \\
\hline $\bar{N}$ & $\overline{32}$ & $\overline{32}$ & 64 & 64 & 128 & 128 & \\
\hline $\bar{\varpi}$ & 0.43 & $\overline{0.40}$ & 0.15 & 0.15 & 0.0038 & 0.0029 & total \\
\hline time & 2.2 & 2.0 & 29.0 & 1.2 & 20.0 & 23.2 & 78 \\
\hline
\end{tabular}

\begin{tabular}{|c|c|c|c|c|}
\hline $\bar{h}$ & 16 & 16 & $\overline{16}$ & \\
\hline $\bar{N}$ & 32 & 64 & 128 & \\
\hline$\varpi$ & 1.40 & 0.86 & 0.69 & total \\
\hline time & 14.0 & 27.1 & 129.8 & 171 \\
\hline
\end{tabular}

\begin{tabular}{|c|c|c|c|c|c|}
\hline$h$ & 128 & 64 & 32 & 16 & \\
\hline $\bar{N}$ & 128 & 128 & 128 & 128 & \\
\hline$\varpi$ & 0.145 & 0.0043 & 0.0038 & 0.0031 & total \\
\hline time & 164.9 & 101.6 & 24.6 & 34.4 & 326 \\
\hline
\end{tabular}

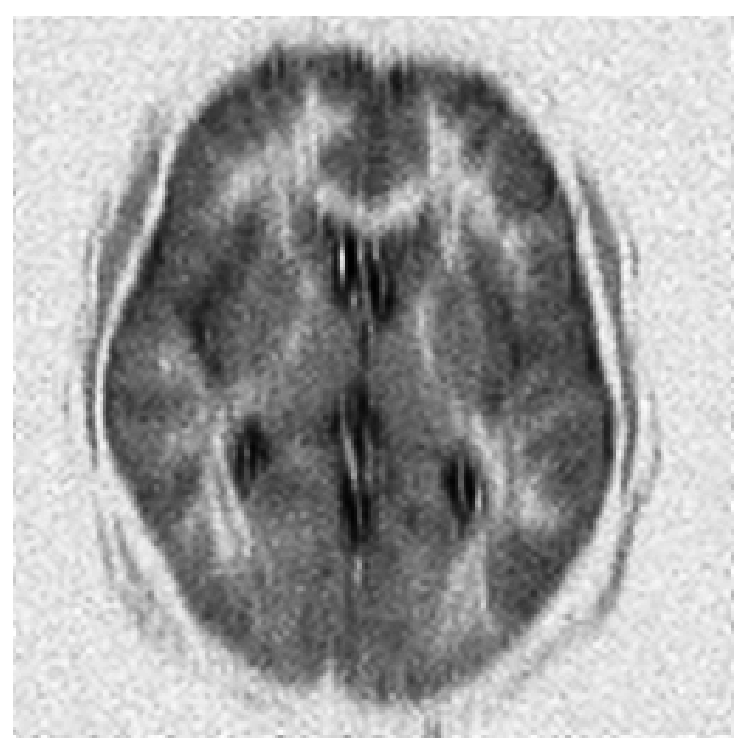

Fig. 8. Artificial EPI image

ceed by registering them with corresponding geometrically correct anatomical MRI images. We have developed a fully automatic image registration algorithm specialized for this task. Our technique increases the geometrical precision of EPI images and thus improves the quality of information obtainable from these images. This will allow the use of EPI images in many clinical and diagnostic applications where they could not have been used previously, as well as an increase of their usefulness in existing applications.

As an additional benefit, our method can be extended to compensate for other causes of geometrical distortion of EPI images besides imperfect magnetic field, such as heart beat and respiration. (In this case, we would look for bidirectional warping.)
The novelty of our registration algorithm stems from a high-order spline model for the warping. It has good approximation properties and lends itself well to a multiresolution approach, while permitting an efficient implementation. We have also taken advantage of a spline model for the image being warped, leading to a second dimension of the multiresolution strategy, and yielding additional computational savings. Finally, we have replaced the customary regularization criterion by a scale parameter of the search space.

We have also presented many experiments to demonstrate the performance of our algorithm. ${ }^{6}$ We plan to carry

\footnotetext{
${ }^{6}$ An online demonstration of our algorithm is available on our WEB page 'http://bigwww.epfl.ch/'.
} 

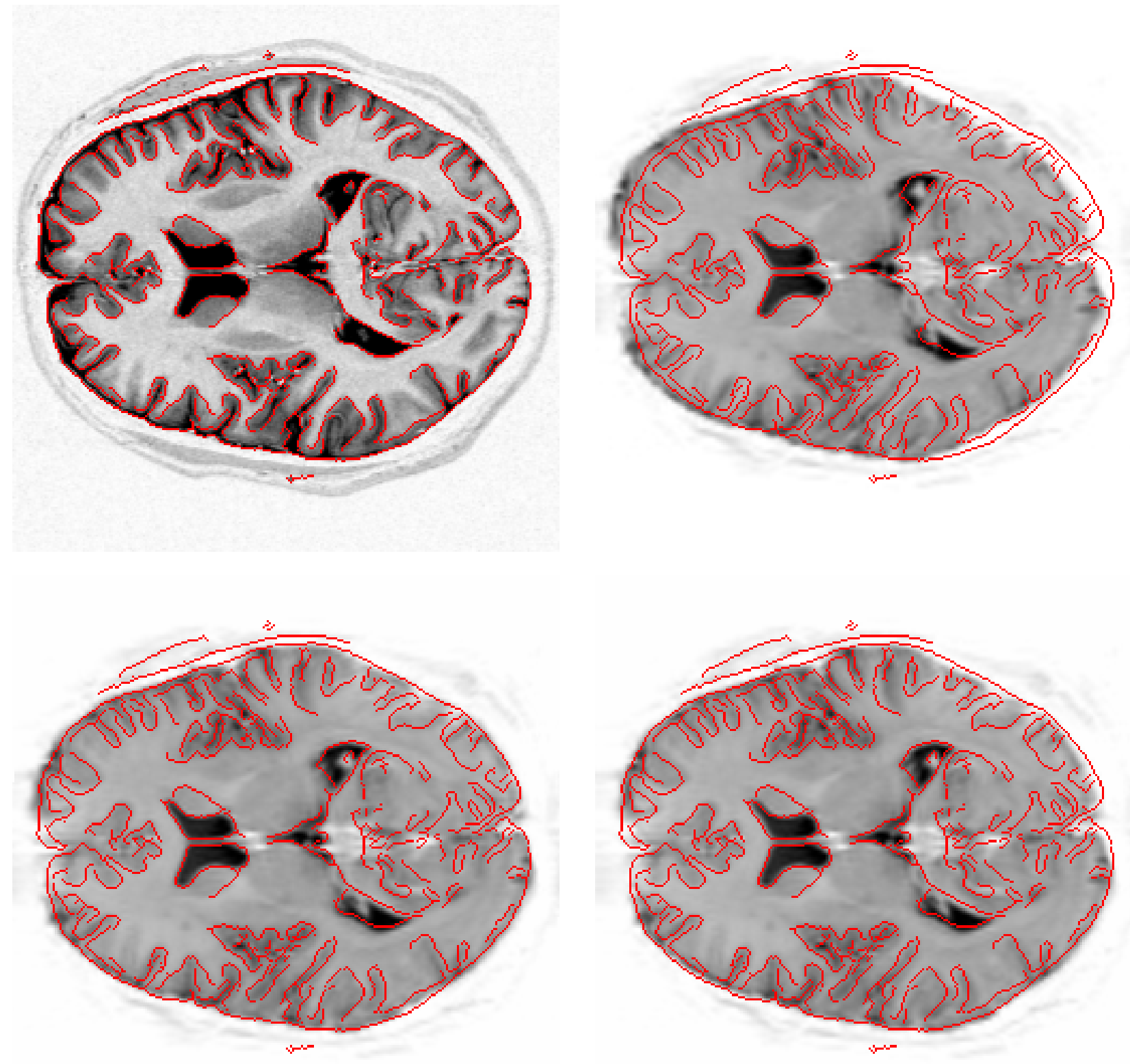

Fig. 9. Anatomical (top-left) and EPI (top-right) images before registration, with superimposed contours from the anatomical images. EPI image after automatic (bottom-left) and manual (bottom-right) registration.

out more clinical experiences to prove conclusively the potential of our method in a real-world setting.

\section{Acknowledgment}

We thank the anonymous reviewers for bringing to our attention a recent conference report [42], which describes a similar approach to ours and that was published after this manuscript had been submitted, at about the same time as our own conference report [43].

\section{APPENDIX}

\section{A. Optimality of the Cubic Spline Model}

The landmark fitting from Section II-B is mathematically equivalent to the smoothing spline problem frequently encountered in statistics [44]. We present here an informal derivation of the variational property of cubic splines. We use an original Fourier-based technique which is instructive and concise.

Consider the following approximation problem: Given a set $\left\{\left(x_{i}, y_{i}\right) \in \mathbb{R}^{2}\right\}$ and a function $\mathcal{L}: \mathbb{R}^{2} \rightarrow \mathbb{R}$, find 
a function $g: \mathbb{R} \rightarrow \mathbb{R}$ minimizing the functional criterion

$$
\begin{aligned}
J^{\prime}(g)=J(g)+\sum_{i} \mathcal{L}\left(g\left(x_{i}\right), y_{i}\right) & \\
\text { with } \quad J(g) & =\int\left|g^{\prime \prime}(x)\right|^{2} \mathrm{~d} x
\end{aligned}
$$

It is not difficult to show that the solution of this problem belongs to the same class as a solution of the interpolation problem, that would consist of finding $g$ minimizing $J(g)$ under the constraints $g\left(x_{i}\right)=z_{i}$, equivalent to landmark interpolation from Section II-B. We will therefore concentrate on the interpolation case here.

Using the Lagrange multiplier method, we construct an augmented criterion

$$
J_{\lambda}=\int\left|g^{\prime \prime}(x)\right|^{2} \mathrm{~d} x-24 \sum_{i} \lambda_{i}\left(g\left(x_{i}\right)-z_{i}\right)
$$

We then express $J_{\lambda}$ in terms of $\hat{g}(\omega)$, the Fourier transform of $g$

$$
\begin{aligned}
J_{\lambda}=\frac{1}{2 \pi} \int \omega^{4}|\hat{g}(w)|^{2} \mathrm{~d} \omega- & \\
& -24 \sum_{i} \lambda_{i}\left(\frac{1}{2 \pi} \int \overline{\hat{g}(\omega)} \mathrm{e}^{-j \omega x_{i}} \mathrm{~d} \omega-z_{i}\right)
\end{aligned}
$$

where we have used $\hat{g}(\omega)=\overline{\hat{g}(-\omega)}$. To find an optimal $\hat{g}(\omega)$, we differentiate $J_{\lambda}$ with respect to $\overline{\hat{g}(\omega)}$ and we impose the first order change to be zero:

$$
\begin{aligned}
\Delta J_{\lambda}= & 2 \operatorname{Re}\left(\frac { 1 } { 2 \pi } \int \overline { \Delta \hat { g } } \left(\hat{g} \omega^{4}-\right.\right. \\
& \left.\left.-12 \sum_{i} \lambda_{i} \mathrm{e}^{-j \omega x_{i}}\right) \mathrm{~d} \omega\right)=0 \quad \text { for all } \overline{\Delta \hat{g}(\omega)}
\end{aligned}
$$

This gives

$$
\hat{g}=\sum_{i} \lambda_{i} \frac{12 \mathrm{e}^{-j \omega x_{i}}}{\omega^{4}}
$$

By interpreting $\mathrm{e}^{-j \omega x_{i}}$ as a phase shift, we get

$$
g(x)=\sum_{i} \lambda_{i} \varrho\left(x-x_{i}\right)
$$

where $\varrho$ is the inverse Fourier transform of $12 / \omega^{4}$, which gives a general form of all solutions to our interpolation or approximation problems as

$$
g(x)=\sum_{i} \lambda_{i}\left|x-x_{i}\right|^{3}
$$

The basis function $|x|^{3}$ is twice continuously differentiable and piecewise cubic. Hence, $g$ is a cubic spline. In order to be complete, this solution needs to be augmented by a linear term generating the null space of $J$. For a rigorous treatment and generalization to multiple dimensions, we refer to [38].
B. B-SPLinES

A B-spline $\beta_{r}$ of degree $r$ is recursively defined as

$$
\begin{array}{r}
\beta_{r}=\beta_{r-1} * \beta_{0} \quad \text { for } r>0 \\
\beta_{0}(x)= \begin{cases}1 & \text { if } x \in\left(-\frac{1}{2}, \frac{1}{2}\right) \\
0 & \text { otherwise }\end{cases}
\end{array}
$$

B-splines as defined above have a compact support $(-r / 2-1 / 2, r / 2+1 / 2)$, are symmetric, and are $(r-1)$-times continuously differentiable. Specifically,

$$
\beta_{3}(x)= \begin{cases}2 / 3-(1-|x| / 2) x^{2} & \text { if } 0<|x| \leq 1 \\ (2-|x|)^{3} / 6 & \text { if } 1<|x|<2 \\ 0 & \text { otherwise }\end{cases}
$$

\section{Explicit Derivatives}

Given $E$ by (4),(6), and (8), let us express the components of $\nabla_{c} E$ and $\nabla_{c}^{2} E$.

$$
\begin{aligned}
\frac{\partial E}{\partial c_{\mathbf{k}}} & =2 \sum_{\mathbf{i} \in I} e_{\mathbf{i}} \frac{\partial e_{\mathbf{i}}}{\partial c_{\mathbf{k}}} \quad \text { where } \quad e_{i}={ }^{c} f(\mathbf{g}(\mathbf{i}))-f^{r}(\mathbf{i}) \\
\frac{\partial e_{\mathbf{i}}}{\partial c_{\mathbf{k}}} & =\left.\left.\frac{\partial^{c} f}{\partial x}\right|_{\mathbf{x}=\mathbf{g}(\mathbf{i})} \cdot \frac{\partial g}{\partial c_{\mathbf{k}}}\right|_{\mathbf{x}=\mathbf{i}}= \\
& =\boldsymbol{\beta}_{3}(\mathbf{i} / h-\mathbf{k}) \sum_{q} b_{q j} \beta_{3}^{\prime}(g(\mathbf{i})-q)
\end{aligned}
$$

where $\mathbf{i}=(i, j)$, and the summation across $q$ only needs to be performed within the support of $\beta_{3}^{\prime}$ of length 4 . The second derivatives are

$$
\begin{aligned}
& \frac{\partial^{2} E}{\partial c_{\mathbf{k}} \partial c_{\mathbf{l}}}=2 \sum_{\mathbf{i} \in I}\left(\frac{\partial e_{\mathbf{i}}}{\partial c_{\mathbf{k}}} \frac{\partial e_{\mathbf{i}}}{\partial c_{\mathbf{l}}}+e_{\mathbf{i}} \frac{\partial^{2} e_{\mathbf{i}}}{\partial c_{\mathbf{k}} \partial c_{\mathbf{l}}}\right) \\
& \frac{\partial^{2} e_{\mathbf{i}}}{\partial c_{\mathbf{k}} \partial c_{\mathbf{l}}}=\left.\frac{\partial^{2} c f}{\partial^{2} x}\right|_{\mathbf{x}=\mathbf{g}(\mathbf{i})} \frac{\partial g}{\partial c_{\mathbf{k}}} \frac{\partial g}{\partial c_{\mathbf{l}}}+\frac{\partial^{c} f}{\partial x} \underbrace{\frac{\partial^{2} g}{\partial c_{\mathbf{k}} \partial c_{\mathbf{l}}}}_{0} \\
& \frac{\partial^{2} e_{\mathbf{i}}}{\partial c_{\mathbf{k}} \partial c_{\mathbf{l}}}=\boldsymbol{\beta}_{3}(\mathbf{i} / h-\mathbf{k}) \boldsymbol{\beta}_{3}(\mathbf{i} / h-\mathbf{l}) \sum_{q} b_{q j} \beta_{3}^{\prime \prime}(g(\mathbf{i})-q)
\end{aligned}
$$

which is easy to calculate because many terms can be reused from the calculation of the first derivatives.

\section{REFERENCES}

[1] Z.-H. Cho, J. P. Jones, and M. Singh, Foundations of Medical Imaging. John Wiley \& Sons, 1993.

[2] X. Wan, G. T. Gullberg, D. L. Parker, and G. L. Zeng, "Reduction of geometric and intensity distortions in echo-planar imaging using a multireference scan," Magnetic Resonance in Medicine, vol. 37, no. 6, pp. 932-942, 1997.

[3] P. Thévenaz, U. E. Ruttimann, and M. Unser, "A pyramid approach to subpixel registration based on intensity," IEEE Transactions on Image Processing, vol. 7, pp. 1-15, Jan. 1998. 
[4] H. Chang and J. M. Fitzpatrick, "A technique for accurate magnetic resonance imaging in the presence of field inhomogeneities," IEEE Transactions on Medical Imaging, vol. 11, pp. 319-329, 1992

[5] B. B. Biswal and J. S. Hyde, "Contour-based registration technique to differentiate between task-activated and head motioninduced signal variations in fMRI," Magnetic Resonance in Medicine, vol. 38, no. 3, pp. 470-476, 1997.

[6] A. L. Alexander, J. S. Tsuruda, and D. L. Parker, "Elimination of Eddy current artifacts in diffusion-weighted echo-planar images: The use of bipolar gradients," Magnetic Resonance in Medicine, vol. 38, no. 6, pp. 1016-1021, 1997.

[7] P. Jezzard and R. S. Balaban, "Correction for geometric distortion in echo planar images from $B_{0}$ field variations," Magnetic Resonance in Medicine, no. 34, pp. 65-73, 1995.

[8] K. Rohr, H. S. Stiehl, R. Sprengel, W. Beil, T. M. Buzug, J. Weese, and M. H. Kuhn, "Point-based elastic registration of medical image data using approximating thin-plate splines," in Visualization in Biomedical Computing (K. H. Höhne and R. Kikinis, eds.), pp. 297-306, Springer-Verlag, 1996.

[9] B. Horn and B. Schunck, "Determining optical flow," Artificial Inteligence, vol. 17, pp. 185-203, 1981.

[10] G. Christensen, Deformable Shape Models for Anatomy. PhD thesis, Washington University, Saint Louis, Mississippi, 1994.

[11] G. Christensen, S. Joshi, and M. Miller, "Volumetric transformation of brain anatomy," IEEE Transactions on Medical Imaging, vol. 16, Dec. 1997.

[12] M. Bro-Nielsen and C. Gramkow, "Fast fluid registration of medical images," in Visualization in Biomedical Computing (K. H. Höhne and R. Kikinis, eds.), pp. 267-276, Springer-Verlag, 1996.

[13] R. Bajcsy and S. Kovačič, "Multiresolution elastic matching," Computer Vision, Graphics, and Image Processing, vol. 46, pp. 1-21, 1989.

[14] D. V. Iosifescu, M. E. Shenton, S. K. Warfield, R. Kikinis, J. Dengler, F. A. Jolesz, and R. W. McCarley, "An automated registration algorithm for measuring MRI subcortical brain structures," Neuroimage, no. 6, pp. 14-25, 1997.

[15] Y. Tai, K. Lin, C. Hoh, S. Huang, and E. Hoffman, "Utilization of 3D elastic transformations in the registration of chest X-ray CT and whole body PET," IEEE Transactions on Nuclear Science, vol. 44, Aug. 1997.

[16] T. Schormann, S. Henn, and K. Zilles, "A new approach to fast elastic alignment with applications to human brains," in Visualization in Biomedical Computing (K. H. Höhne and R. Kikinis, eds.), Springer-Verlag, 1996.

[17] J. Bergen, P. Anandan, K. Hanna, and R. Hingorani, "Hierarchical model-based motion estimation," in Second European Conference on Computer Vision (ECCV'92), pp. 237-252, SpringerVerlag, 1992.

[18] N. Sicotte, R. Woods, and J. Mazziotta, "Automated image registration using a 105 parameter non-linear model," Neuroimage, vol. 3, June 1996. Second International Conference on Functional Mapping of the Human Brain.

[19] R. Woods, S. Grafton, N. Sicotte, and J. Mazziotta, "Automated image registration: II. Intersubject validation of linear and nonlinear models," Journal of Computer Assisted Tomography, vol. 22, no. 1, pp. 153-165, 1998.

[20] S. Kiebel, J. Ashburner, J. Poline, and K. Friston, "MRI and PET coregistration - a cross validation of statistical parametric mapping and automated image registration," Neuroimage, no. 5, 1997.

[21] A. W. Toga, ed., Brain Warping. San Diego: Academic Press, 1999.

[22] P.Thompson and A. W. Toga, "A surface-based technique for warping 3-dimensional images of the brain," IEEE Transactions on Medical Imaging, vol. 4, pp. 1-16, Aug. 1996.

[23] C. Davatzikos, "Spatial normalization of 3D brain images using deformable models," Journal of Computer Assisted Tomography, vol. 4, no. 20, pp. 656-665, 1996.

[24] J. Declerck, G. Subsol, J. P. Thirion, and N. Ayache, "Automatic retireval of anatomical structuires in 3d medical images," in CVRMed'95, Lecture Notes in Computer Science (N. Ayache, ed.), pp. 153-162, Nice, France: Springer-Verlag, Apr. 1995.

[25] F. Bookstein, Morphometric Tools for Landmark Data: Geometry and Biology. Cambridge University Press, 1997.

[26] B. Kim, J. Boes, K. Frey, and C. Meyer, "Mutual information for automated multimodal image warping," in Visualization in Biomedical Computing (K. H. Höhne and R. Kikinis, eds.), Springer-Verlag, 1996.

[27] H. Lester and S. Arridge, "Summarising fluid registration by thin-plate spline warps with many landmarks," in Medical image Understanding and Analysis, July 1997. http://www.robots.ox.ac.uk/ mvl/miua97/.

[28] M. Unser, A. Aldroubi, and M. Eden, "B-spline signal processing: Part I-theory," IEEE Transactions on Signal Processing, vol. 41, pp. 821-832, Feb. 1993.

[29] M. Unser, A. Aldroubi, and M. Eden, "B-spline signal processing: Part II-efficient design and applications," IEEE Transactions on Signal Processing, vol. 41, pp. 834-848, Feb. 1993.

[30] J. Ahlberg, E. Nilson, and J. Walsh, The Theory of Splines and Their Applications. New York: Academic Press, 1967.

[31] I. Schoenberg, "Spline functions and the problem of graduation," Proc. Nat. Acad. Sci., vol. 52, pp. 947-950, 1964.

[32] M. Unser and T. Blu, "Fractional splines and wavelets," SIAM Review, 1999. in print.

[33] T. Blu and M. Unser, "Quantitative Fourier analysis of approximation techniques: Part I-interpolators and projectors," IEEE Transactions on Signal Processing, 1999.

[34] M. Unser, A. Aldroubi, and M. Eden, "Fast B-spline transforms for continuous image representation and interpolation," IEEE Trans. Pattern Anal. Mach. Intell., vol. 13, Mar. 1991.

[35] G. Subsol, Construction automatique d'atlas anatomiques morphométriques à partir d'images médicales tridimensionnelles. PhD thesis, Ecole Centrale, Paris, France, 1995.

[36] W. H. Press, S. A. Teukolsky, W. T. Vetterling, and B. P. Flannery, Numerical Recipes in C. Cambridge University Press, second ed., 1992.

[37] F. Müller, P. Brigger, K. Illgner, and M. Unser, "Multiresolution approximation using shifted splines," IEEE Transactions on Signal Processing, vol. 46, pp. 2555-2558, Sept. 1998.

[38] J. Duchon, "Splines minimizing rotation-invariant semi-norms in Sobolev spaces," in Constructive Theory of Functions of Several Variables (W. Schempp and K. Zeller, eds.), (Berlin), pp. 85100, Springer-Verlag, 1977.

[39] S. Mallat, A Wavelet Tour of Signal Processing. San Diego, CA: Academic Press, 1998.

[40] P. Moulin, R. Krishnamurthy, and J. Woods, "Multiscale modeling and estimation of motion fields for video coding," IEEE Transactions on Image Processing, vol. 6, Dec. 1997.

[41] R. Szeliski and J. Coughlan, "Spline-based image registration," International Journal of Computer Vision, vol. 22, pp. 199-218, 1997.

[42] C. Studholme, R. T. Constable, and J. S. Duncan, "Incorporating an image distortion model in non-rigid alignment of EPI with conventional MRI," in International Conference on Information Processing in Medical Imaging, Lecture Notes in Computer Science, (Visegràd, Hungary), pp. 454-459, Springer-Verlag, June 1999.

[43] J. Kybic, P. Thévenaz, and M. Unser, "Multiresolution spline warping for EPI registration," in Proceedings of SPIE, vol. 3813, (Denver, Colorado), pp. 571-579, SPIE, July 1999.

[44] G. Wahba, Spline Models for Observational Data. Philadelphia, PA: SIAM, 1990. 